\title{
Voltage-to-Frequency Converter for Low-Power Sensor Interfaces
}

\author{
C. Azcona, B. Calvo, N. Medrano, S. Celma \\ Grupo de Diseño Electrónico (GDE) \\ Instituto de Investigación en Ingeniería de Aragón (I3A) \\ Universidad de Zaragoza, C/ Pedro Cerbuna 12, 50009, Zaragoza, Spain. \\ Tel.+34-976761240, Fax +34-976762143, e-mail \{cris.azcona, becalvo,nmedrano, scelma\}@unizar.es
}

\begin{abstract}
This work presents a low-power rail-to-rail temperature compensated voltage-to-frequency converter (VFC) which constitutes the last stage of a sensor read-out interface targeting wireless sensor networks (WSN) applications. These quasi-digital converters are now receiving great interest, since they combine the simplicity of analog devices with the accuracy and noise immunity proper to digital signal processing; besides, frequency output is directly driven to the embedded node microcontroller $\mu \mathrm{C}$, which next performs the $\mathrm{A} / \mathrm{D}$ conversion using its internal timers. A first read-out interface prototype using low-voltage low-power commercial components shows that the VFC means $99 \%$ of the total interface consumption in read-out mode. Further, existing CMOS VFCs in the form of ASICs have a rather limited input range and an unsuitable output frequency span for typical $\mu \mathrm{C}$ clock frequencies used in WSN. Hence, a novel full custom VFC solution is needed, fullfilling the main requirements of rail-to-rail operation, to take advantage of the full supply voltage range to optimize the output frequency resolution, and low-power low-voltage operation to have a power supply compatible with conventional WSN batteries while maximizing the operating life of the sensor node. Experimental results for a $0.18-\mu \mathrm{m}$ 1.2-V CMOS VFC implementation show for an input range of $(0-1.2 \mathrm{~V})$ an output frequency range of $(0.1-1.0 \mathrm{MHz})$, adequate to digitize the signal with the direct counting method in the sensor node $\mu \mathrm{C}$ achieving 13 bits resolution. It has a power consumption of $60 \mu \mathrm{W}$ (35 $\mathrm{nW}$ in sleep mode) and it is temperature insensitive for a temperature range of $\left(-40,120^{\circ} \mathrm{C}\right)$.
\end{abstract}

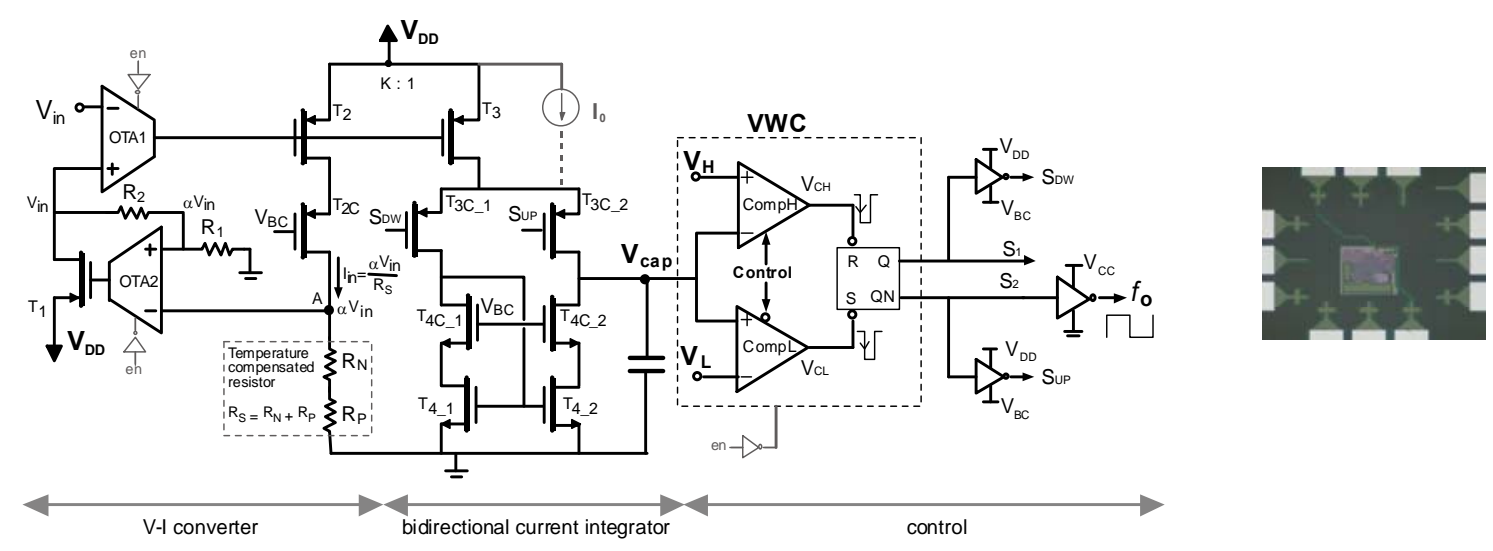

Fig. 1: Basic scheme of the integrated VFC and its microphotograph. 